\title{
HALF-PARABOLIC QUANTUM WELLS OF DILUTED MAGNETIC SEMICONDUCTOR
}

\author{
$\mathrm{Cd}_{1-x} \mathrm{Mn}_{x} \mathrm{Te}^{*}$
}

M. Kutrowski, T. Wojtowicz, G. Cywiński, G. KARCZewski, E. JANIK, E. Dynowska, J. Kossut

Institute of Physics, Polish Academy of Sciences

Al. Lotników 32/46, 02-668 Warsaw, Poland

\section{P. KosSACKI}

Institute of Experimental Physics, Warsaw University

Hoża 69, 00-681 Warsaw, Poland

\section{R. Fiederling, A. Pfeuffer-Jeschike and W. Ossau}

Physikalisches Institut der Universität Würzburg, 97074 Würzburg, Germany

We report on magnetooptical studies of MBE-grown half-parabolic $\mathrm{CdTe} / \mathrm{Cd}_{x} \mathrm{Mn}_{1-x}$ Te quantum well structures. The value of the valence band offset $Q_{v}=0.4 \pm 0.05$ was determined by comparing energies of optical transitions in the absence of a magnetic field with model calculations. This value was verified by fitting the observed spin splitting of the lowest heavy hole (h) state. We discuss also the temperature dependence of $Q_{\mathrm{v}}$.

PACS numbers: 78.66.Hf, 75.50.Pp

Quantum wells (QWs) with parabolically shaped confining potential were found to possess very interesting and unique physical properties. They have also the potential for possible applications [1]. Energy levels in parabolic QWs (PQWs) are very sensitive to the actual valence band offset. The accuracy of the band offset determination from the studies of parabolic quantum wells [2] can be further increased when not only the "diagonal" excitonic transitions (i.e., those with $\Delta n=n_{\mathrm{e}}-n_{\mathrm{hh}}=0$, where $n_{\mathrm{e}}$ and $n_{\mathrm{hh}}$ are confined level quantum numbers in the conduction and heavy holes band, respectively) but also "nondiagonal" $(\Delta n \neq 0)$ transitions are observed. The appearance of transitions with $\Delta n \neq 0$ is to be expected in half-parabolic quantum wells (HPQWs) whose confining potential lacks the reflection symmetry. This leads to an undetermined parity of the states and, in

*This work is supported in part by the Committee for Scientific Research (Poland) through grant 8T11B 01411 and by Volkswagen Foundation. 
consequence, to relaxation of the parity-related selection rules for optical transitions. Thus, in the case of a HPQW, in comparison with a PQW of the same width, one should observe a greater number of optical transitions. The additional advantage of HPQWs grown by MBE by a digital method [3] is that such asymmetric potential shape is easier to render accurately than in the case of $P Q W$.

In this paper we report on growth and magnetooptical studies of half-parabolic quantum wells made of diluted magnetic semiconductor $\mathrm{Cd}_{1-x} \mathrm{Mn}_{x} \mathrm{Te}$. The structures were grown on GaAs (100) oriented substrates using molecular beam epitaxy and the digital growth method which employs Mn flux pulses of different duration $[1,3]$.

In particular, we studied samples that contained either a single HPQW grown on a thick $(3 \mu \mathrm{m})$ CdTe buffer or a multiple HPQW (10 wells separated by 100 monolayers $(\mathrm{ML})=324 \AA$ ) grown directly on GaAs. The samples were studied using either transmission (multiple HPQW, after removal of the nontransparent GaAs substrate) or photoluminescence excitation (PLE) spectroscopy (all samples) in magnetic fields up to $7.5 \mathrm{~T}$ and in the temperature range from $1.6 \mathrm{up}$ to $270 \mathrm{~K}$. The well widths $L_{z}$ and Mn molar fraction $x$ in the outermost region of the barrier of the investigated structures were: $L_{z}=80 \mathrm{ML}=259 \AA, x=0.77$ and $L_{z}=80 \mathrm{ML}, x=0.5$ in single HPQW samples; and $L_{z}=60 \mathrm{ML}$ with $x=0.25$ in the multiple HPQW sample. In all cases CdTe constituted the material of the bottom of well (at $z=0$ ).

Optical spectra obtained in both types of experiments revealed several series of peaks equidistant in energy associated with "diagonal" interband excitonic transitions between harmonic oscillator-like levels in the conduction and heavy- and light-hole bands. Additionally, a "nondiagonal" $\Delta n=1$ transition between $\mathrm{hh}_{2}$ and $e_{1}$ levels was observed in the absence of an external magnetic field. This transition could be unambiguously identified on the basis of the spin splitting, since the magnetic field dependence of energy of the transitions involving the same heavy hole states (e.g. $\mathrm{hh}_{2} \rightarrow \mathrm{e}_{2}$ and $\mathrm{hh}_{2} \rightarrow \mathrm{e}_{1}$ ) are nearly identical. Several additional "nondiagonal" transitions appeared with magnetic field applied. The value of the energy of the transitions extracted from the absorption and from the PLE experiments in the multiple HPQW sample remained in very good agreement.

In the infinite well approximation the interlevel distances $\Delta E_{\mathrm{hh}}$ and $\Delta E_{\mathrm{e}}$ in the heavy hole and conduction bands, respectively, can be described by the following equations:

$$
\Delta E_{\mathrm{hh}}=\frac{2 \hbar}{L_{z}} \sqrt{\frac{2 \Delta E_{\mathrm{g}} Q_{\mathrm{v}}}{m_{\mathrm{hh}}}}, \quad \Delta E_{\mathrm{e}}=\frac{2 \hbar}{L_{z}} \sqrt{\frac{2 \Delta E_{\mathrm{g}}\left(1-Q_{\mathrm{v}}\right)}{m_{\mathrm{e}}}},
$$

where $\Delta E_{\mathrm{g}}$ is the energy gap discontinuity between the materials at $z=0$ and at $z=L_{z}, m_{\mathrm{hh}}^{*}$ and $m_{\mathrm{e}}^{*}$ are effective masses of the heavy holes and electrons, respectively, and $Q_{\mathrm{v}}$ is the valence band offset.

The observation of the "nondiagonal" transitions allows the determination of the interlevel energy distance between the confined states in the conduction and heavy hole bands separately. This, in turn, makes possible an independent determination of the conduction and valence band offsets, provided, of course, that the effective masses of both types of carriers are known. This is in contrast to 

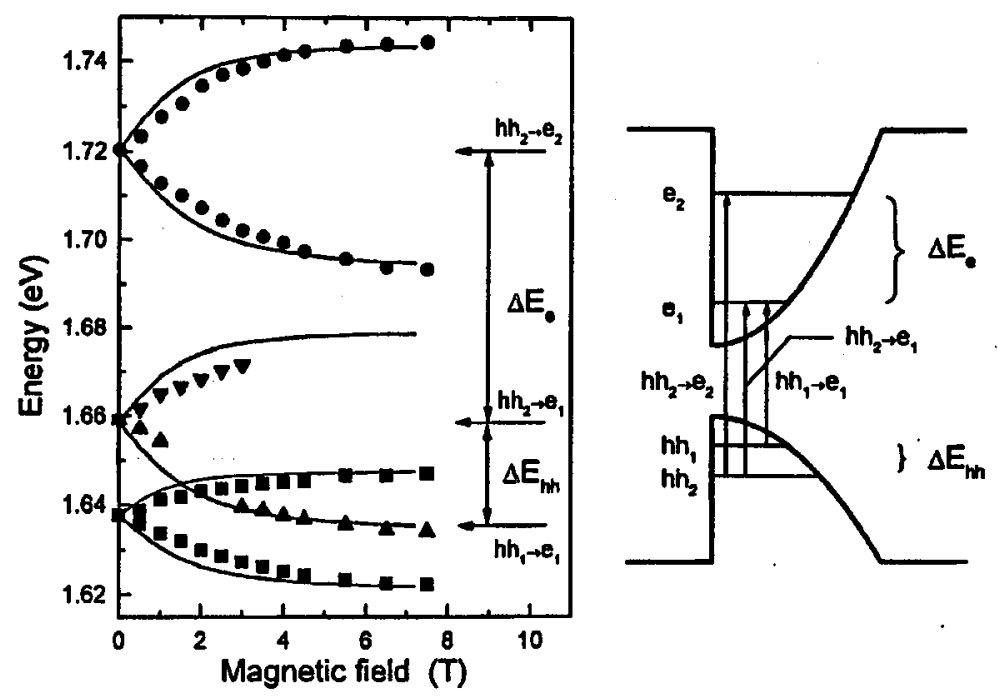

Fig. 1. The energy positions measured by PLE (points) in a single HPQW with $L_{z}=$ $80 \mathrm{ML}, x=0.5$ for three types of transitions: $\mathrm{hh}_{1} \rightarrow \mathrm{e}_{1}, \mathrm{hh}_{2} \rightarrow \mathrm{e}_{2}$ and $\mathrm{hh}_{2} \rightarrow \mathrm{e}_{1}$ as a function of magnetic field. The continuous lines represent the result of numerical calculations for $Q_{\mathrm{v}}=0.40$, with the Luttinger and electron mass parameters as indicated in text. The interlevel distances $\Delta E_{\mathrm{hh}}$ and $\Delta E_{\mathrm{e}}$ are also indicated on the right hand side. The scheme of energy levels and possible transitions in the HPQW system are shown.

the situation in parabolic QWs, where the valence and the conduction band offsets appear jointly in the appropriate equations describing the "diagonal" transitions.

In Fig. 1 we show the measured energy of the transitions (points) versus the external magnetic field strength in a HPQW with $L_{z}=80 \mathrm{ML}$ and $x=0.5$ in the barrier for the three types of transitions: $\mathrm{hh}_{1} \rightarrow \mathrm{e}_{1}, \mathrm{hh}_{2} \rightarrow \mathrm{e}_{2}$ and $\mathrm{hh}_{2} \rightarrow \mathrm{e}_{1}$. The lines represent a model calculation based on the numerical solution of the Luttinger Hamiltonian describing the valence band, and of the conduction band with a quadratic dispersion, with an additional half-parabolic potential shape. The calculations were performed with the following values of the Luttinger parameters: $\gamma_{1}=4.5, \gamma_{2}=1.46$, and with a constant effective mass of electrons, $m_{\mathrm{e}}=0.09 m_{0}$ [4]. Good agreement between calculations and experimental data was obtained in the absence of the magnetic field for $Q_{\mathrm{v}}=0.40$. Similar agreement could be also obtained in the case of the remaining HPQW samples, giving $Q_{v}=0.40 \pm 0.05$, and confirming the previous determination of $Q_{v}=0.40$ [3]. The accuracy is mainly limited by the uncertainty in the effective mass values. This value is not in contradiction with the recent determination of $Q_{\mathrm{v}}$ by Leibihen et al. [5].

For the calculation of the magnetic field dependence of the transition energy we assumed that the quantum well potential shape is determined by the bulk Zeeman splitting of $\mathrm{Cd}_{1-x} \mathrm{Mn}_{x} \mathrm{Te}$. We used here the parametrization of the Zeeman splitting given in Ref. [6]. As can be seen in Fig. 1, the calculations describe 
the splitting of $h h_{1} \rightarrow e_{1}$ transition quite well, particularly at saturation of the magnetization. Similar agreement can be obtained for transitions involving the $\mathrm{hh}_{2}$ state. $Q_{v}$ extracted from the analysis of the spin splitting of $h_{h_{1}} \rightarrow \mathrm{e}_{1}$ transition is in agreement with that obtained above from the analysis of the $h_{h} \rightarrow e_{1}$, $\mathrm{hh}_{2} \rightarrow \mathrm{e}_{2}$ and $\mathrm{hh}_{2} \rightarrow \mathrm{e}_{1}$ transitions at $B=0$. This again confirms that the shape of the QW at $B=0$ is in fact half-parabolic.

We performed also the measurements of the transmission spectra for the $60 \mathrm{ML}$ multiple HPQW at temperatures up to $270 \mathrm{~K}$ in the absence of the field. A very rapid decrease in intensity of the "nondiagonal" transition was observed. It disappears at about $15 \mathrm{~K}$, while the "diagonal" transitions were observed up to $250 \mathrm{~K}$. Thus the direct determination of $Q_{\mathrm{v}}$ was not possible for temperatures exceeding $15 \mathrm{~K}$. However, a preliminary analysis of the temperature dependence of the transition energies shows that, within experimental error, there is no change in the value of $Q_{v}$ with temperature.

\section{References}

[1] A.C. Gossard, M. Sundaram, P.F. Hopkins, in: Epitaxial Microstructures, in series Semiconductors and Semimetals, Vol. 40, Ed. A.C. Gossard, Academic Press, Boston 1994, p. 153.

[2] T. Wojtowicz, M. Kutrowski, G. Cywinski, E. Dynowska, G. Karczewski, J. Kossut, R. Fiederling, G. Mackh, U. Zehnder, W. Ossau, Acta Phys. Pol. A 90, 977 (1996).

[3] T. Wojtowicz, M. Kutrowski, M. Surma, K. Kopalko, G. Karczewski, J. Kossut, M. Godlewski, Appl. Phys. Lett. 68, 3326 (1996).

[4] Le Si Dang, R. Romestain, Solid State Commun. 44, 1187 (1982).

[5] T. Leibihen, E. Deleporte, C. Delalande, Phys. Rev. B 55, 1724 (1997).

[6] J.A. Gaj, W. Grieshaber, C. Bodin-Deshayes, J. Cibert, G. Feuillet, Y. Merle d'Aubigné, A. Wasiela, Phys. Rev. B 50, 5512 (1994). 